\title{
Nucleation in the Chiral Transition with an Inhomogeneous Background
}

\author{
Bruno G. Taketani and Eduardo S. Fraga \\ Instituto de Física, Universidade Federal do Rio de Janeiro \\ C.P. 68528, Rio de Janeiro, RJ 21941-972, Brazil
}

Received on 19 January, 2007

\begin{abstract}
We consider an approximation procedure to evaluate the finite-temperature one-loop fermionic density in the presence of a chiral background field which systematically incorporates effects from inhomogeneities in the chiral field through a derivative expansion. Modifications in the effective potential and their consequences for the bubble nucleation process are discussed.
\end{abstract}

Keywords: Chiral symmetry breaking

The mechanism of chiral symmetry breaking and the study of the dynamics of phase conversion after a temperaturedriven chiral transition can be done conveniently within lowenergy effective models [1-9]. In particular, to study the mechanisms of bubble nucleation and spinodal decomposition in a hot expanding plasma [10], it is common to adopt the linear $\sigma$-model coupled to quarks [11]. The gas of quarks provides a thermal bath in which the long-wavelength modes of the chiral field evolve, and the latter plays the role of an order parameter in a Landau-Ginzburg approach to the description of the chiral phase transition. The standard procedure is then integrating over the fermionic degrees of freedom, using a classical approximation for the chiral field, to obtain a formal expression for the thermodynamic potential from which one can obtain all the physical quantities of interest.

To compute correlation functions and thermodynamic quantities, one has to evaluate the fermionic determinant that results from the functional integration over the quark fields within some approximation scheme. Usually one performs a standard one-loop calculation assuming a homogeneous and static background field [12]. Nevertheless, for a system that is in the process of phase conversion after a chiral transition, one expects inhomogeneities in the chiral field configuration due to fluctuations to play a major role in driving the system to the true ground state. In fact, for high-energy heavy ion collisions, hydrodynamical studies have shown that significant density inhomogeneities may develop dynamically when the chiral transition to the broken symmetry phase takes place [6], and inhomogeneities generated during the late stages of the nonequilibrium evolution of the order parameter might leave imprints on the final spatial distributions and even on the integrated, inclusive abundances [13].

In this paper we briefly describe an approximation procedure to evaluate the finite-temperature fermionic density in the presence of a chiral background field, which systematically incorporates effects from inhomogeneities in the bosonic field through a gradient expansion. (For details, see Ref. [14].) The method is valid for the case in which the chiral field varies smoothly, and allows one to extract information from its long-wavelength behavior, incorporating corrections order by order in the derivatives of the field. For simplicity, we ignore corrections coming from bosonic fluctuations in what follows. Those would result in a bosonic determinant correction to the effective potential [15]. To focus on the effect of an inhomogeneous background field on the fermionic density, we treated the scalar field essentially as a "heavy" (classical) field, whereas fermions were assumed to be "light".

Let us consider a scalar field $\phi$ coupled to fermions $\psi$ according to the Lagrangian

$$
\mathcal{L}=\bar{\psi}\left[i \gamma^{\mu} \partial_{\mu}+\mu \gamma^{0}-M(\phi)\right] \psi+\frac{1}{2} \partial_{\mu} \phi \partial^{\mu} \phi-V(\phi),
$$

where $\mu$ is the fermionic chemical potential, $M(\phi)$ is the effective mass of the fermions and $V(\phi)$ is a self-interaction potential for the bosonic field.

In the case of the linear $\sigma$-model coupled to quarks, $\phi$ represents the $\sigma$ direction of the chiral field $\Phi=(\sigma, \vec{\pi})$, where $\pi^{i}$ are pseudoscalar fields playing the role of the pions, which we drop here for simplicity. The field $\psi$ plays the role of the constituent-quark field $q=(u, d)$, and $\mu=\mu_{q}$ is the quark chemical potential. The "effective mass" is given by $M(\phi)=$ $g|\sigma|$, and $V(\Phi)=\left(\lambda^{2} / 4\right)\left(\sigma^{2}+\vec{\pi}^{2}-v^{2}\right)^{2}-h_{q} \sigma$ is the selfinteraction potential for $\Phi$. The parameters above are chosen such that chiral $S U_{L}(2) \otimes S U_{R}(2)$ symmetry is spontaneously broken in the vacuum. The vacuum expectation values of the condensates are $\langle\sigma\rangle=f_{\pi}$ and $\langle\vec{\pi}\rangle=0$, where $f_{\pi}=93 \mathrm{MeV}$ is the pion decay constant. The explicit symmetry breaking term is due to the finite current-quark masses and is determined by the PCAC relation, giving $h_{q}=f_{\pi} m_{\pi}^{2}$, where $m_{\pi}=138 \mathrm{MeV}$ is the pion mass. This yields $v^{2}=f_{\pi}^{2}-m_{\pi}^{2} / \lambda^{2}$. The value of $\lambda^{2}=20$ leads to a $\sigma$-mass, $m_{\sigma}^{2}=2 \lambda^{2} f_{\pi}^{2}+m_{\pi}^{2}$, equal to $600 \mathrm{MeV}$.

The Euler-Lagrange equation for static chiral field configurations contains a term which represents the fermionic density:

$$
\nabla^{2} \phi=\frac{\partial V}{\partial \phi}+g \rho(T, \mu, \phi)
$$

and the density of fermions at a given point $\vec{x}_{0}$ has the form

$$
\rho\left(\vec{x}_{0}\right)=\operatorname{Sp}\left\langle\vec{x}_{0}\left|\frac{1}{G_{E}^{-1}+M(\hat{x})}\right| \vec{x}_{0}\right\rangle,
$$

where $\left|\vec{x}_{0}\right\rangle$ is a position eigenstate with eigenvalue $\vec{x}_{0}$, and $S p$ represents a trace over fermionic degrees of freedom, such as color, spin and isospin. 
In order to take into account inhomogeneity effects of the chiral background field, $\phi$, encoded in the position dependence of $M$ in (3), we resort to a derivative expansion as explained below [14].

In momentum representation, the expression for the fermionic density assumes the form

$\rho\left(\vec{x}_{0}\right)=\operatorname{SpT} \sum_{n} \int \frac{d^{3} k}{(2 \pi)^{3}} e^{-i \vec{k} \cdot \vec{x}} \frac{1}{\gamma^{0}\left(i \omega_{n}+\mu\right)-\vec{\gamma} \cdot \vec{k}+M(\hat{x})} e^{i \vec{k} \cdot \vec{x}}$.

One can transfer the $\vec{x}_{0}$ dependence to $M(\hat{x})$ through a unitary transformation, obtaining

$$
\rho\left(\vec{x}_{0}\right)=\operatorname{Sp} T \sum_{n} \int \frac{d^{3} k}{(2 \pi)^{3}} \frac{1}{\gamma^{0}\left(i \omega_{n}+\mu\right)-\vec{\gamma} \cdot \vec{k}+M\left(\hat{x}+\vec{x}_{0}\right)},
$$

where one should notice that $\vec{x}_{0}$ is a c-number, not an operator.

Now we expand $M\left(\hat{x}+\vec{x}_{0}\right)$ around $\vec{x}_{0}$ :

$$
\begin{aligned}
M\left(\hat{x}+\vec{x}_{0}\right) \equiv & M\left(\vec{x}_{0}\right)+\Delta M\left(\hat{x}, \vec{x}_{0}\right)= \\
& M\left(\vec{x}_{0}\right)+\nabla_{i} M \hat{x}^{i}+\frac{1}{2} \nabla_{i} \nabla_{j} M \hat{x}^{i} \hat{x}^{j}+\cdots
\end{aligned}
$$

and use $\hat{x}^{i}=-i \nabla_{k_{i}}$ to write

$$
\rho\left(\vec{x}_{0}\right)=\operatorname{Sp} T \sum_{n} \int \frac{d^{3} k}{(2 \pi)^{3}} \frac{1}{\gamma^{0}\left(i \omega_{n}+\mu\right)-\vec{\gamma} \cdot \vec{k}+M\left(\vec{x}_{0}\right)}\left[1+\Delta M\left(-i \nabla_{k_{i}}, \vec{x}_{0}\right) \frac{1}{\gamma^{0}\left(i \omega_{n}+\mu\right)-\vec{\gamma} \cdot \vec{k}+M\left(\vec{x}_{0}\right)}\right]^{-1} .
$$

To study the dynamics of phase conversion after a chiral transition, one can focus on the long-wavelength properties of the chiral field. From now on we assume that the static background, $M(\vec{x})$, varies smoothly and fermions transfer a small ammount of momentum to the chiral field. Under this assumption, we can expand the expression inside brackets in Eq. (7) in a power series:

$$
\rho(\vec{x})=\operatorname{SpT} \sum_{n} \int \frac{d^{3} k}{(2 \pi)^{3}} \frac{1}{\gamma^{0}\left(i \omega_{n}+\mu\right)-\vec{\gamma} \cdot \vec{k}+M(\vec{x})} \sum_{\ell}(-1)^{\ell}\left[\Delta M\left(-i \nabla_{k_{i}}, \vec{x}\right) \frac{1}{\gamma^{0}\left(i \omega_{n}+\mu\right)-\vec{\gamma} \cdot \vec{k}+M(\vec{x})}\right]^{\ell}
$$

Eq. (8), together with

$\Delta M\left(-i \nabla_{k_{i}}, \vec{x}\right)=\nabla_{i} M\left(\frac{1}{i}\right) \nabla_{k_{i}}+\frac{1}{2} \nabla_{i} \nabla_{j} M\left(\frac{1}{i}\right)^{2} \nabla_{k_{i}} \nabla_{k_{j}}+\cdots$,

provides a systematic procedure to incorporate corrections brought about by inhomogeneities in the chiral field to the quark density, so that one can calculate $\rho(\vec{x})=\rho_{0}(\vec{x})+\rho_{1}(\vec{x})+$ $\rho_{2}(\vec{x})+\cdots$ order by order in powers of the derivative of the background, $M(\vec{x})$.

The leading-order term in this gradient expansion for $\rho(\vec{x})$ can be calculated in the standard fashion [12] and yields the well-known mean field result for the scalar quark density

$$
\rho_{0}=v_{q} \int \frac{d^{3} k}{(2 \pi)^{3}} \frac{M(\phi) / E_{k}(\phi)}{e^{\left[E_{k}(\phi)-\mu_{q}\right] / T}+1}+\left(\mu_{q} \rightarrow-\mu_{q}\right),
$$

where $v_{q}=12$ is the color-spin-isospin degeneracy factor, $E_{k}(\phi)=\left(\vec{k}^{2}+M^{2}(\phi)\right)^{1 / 2}$, and $M(\phi)=g|\phi|$ plays the role of an effective mass for the quarks. The net effect of this leading term is correcting the potential for the chiral field, so that we can rewrite Eq. (2) as

$$
\nabla^{2} \phi=\frac{\partial V_{e f f}}{\partial \phi}
$$

where $V_{\text {eff }}=V(\phi)+V_{q}(\phi)$ and

$$
V_{q} \equiv-v_{q} T \int \frac{d^{3} k}{(2 \pi)^{3}} \ln \left(e^{\left[E_{k}(\phi)-\mu_{q}\right] / T}+1\right)+\left(\mu_{q} \rightarrow-\mu_{q}\right) .
$$

This kind of effective potential is commonly used as the coarse-grained thermodynamic potential in a phenomenological description of the chiral transition for an expanding quarkgluon plasma [3-6].

The next non-trivial term in the expansion contains two contributions: one coming from $\rho_{1}$ and another from $\rho_{2}$. This is due to the rearrangement of powers of the gradient operator. This term will correct the Laplacian piece in the chiral field 


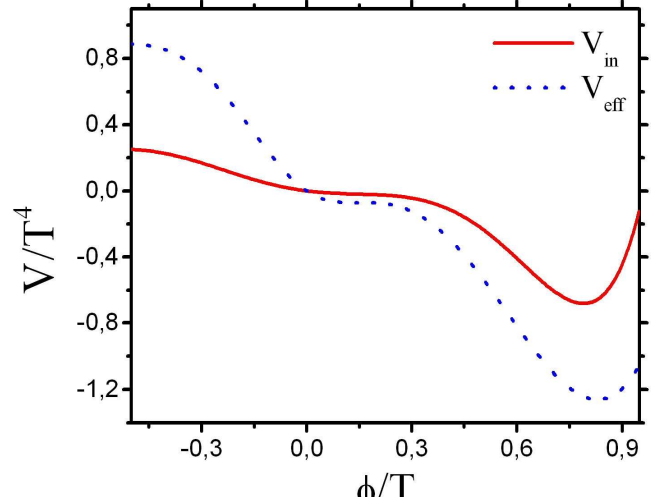

(a) $\phi / \mathrm{T}$

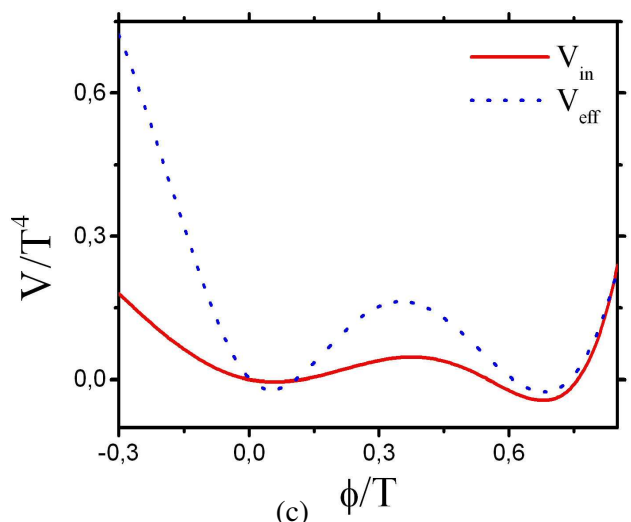

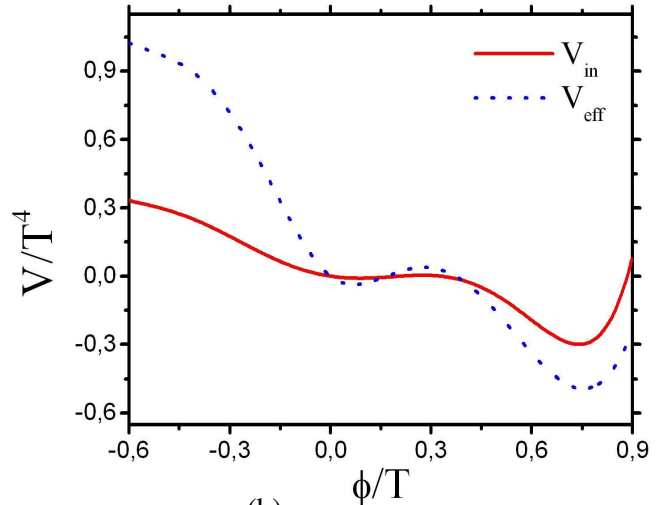

(b)

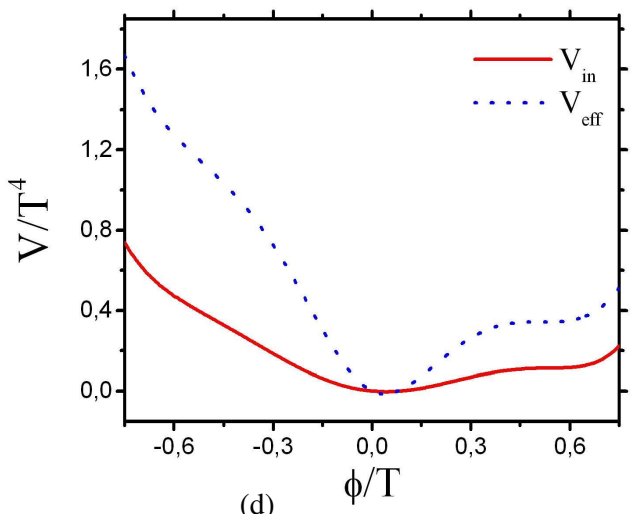

(d)

FIG. 1: $V_{\text {eff }}$ and $V_{\text {in }}(\phi)$ for different values of the temperature $T=[108(\mathrm{a}), 116(\mathrm{~b}), 124(\mathrm{c}), 132(\mathrm{~d})] \mathrm{MeV}$ at $\mu_{q}=0$ and for $g=5.5$.

equation. Dropping zero-temperature contributions which can be absorbed by a redefinition of the bare parameters in $V$, a long but straightforward calculation yields [14]

$$
\left(\rho_{1}+\rho_{2}\right)=-\left(\nabla^{2} M\right) W_{q}\left(T, \mu_{q}, \phi\right),
$$

where

$$
W_{q}\left(T, \mu_{q}, \phi\right)=\frac{v_{q}}{2 \pi^{2}} \int_{0}^{\infty} d k k^{2}\left[H\left(E_{k}, T, \mu_{q}\right)+H\left(E_{k}, T,-\mu_{q}\right)\right],
$$

and $H\left(E_{k}, T, \mu_{q}\right)$ is a complicated function whose particular form is not very illuminating. In the low-temperature limit $(\beta M>>1)$, the integral above is strongly suppressed for high values of $k$, and the leading term has the much simpler form

$$
W_{q}\left(T, \mu_{q}, \phi\right) \approx \frac{v_{q}}{\pi^{2}} \frac{\sqrt{2 \pi}}{8} e^{-\beta|M|}(\beta|M|)^{3 / 2},
$$

which gives a a better idea of the profile of the first inhomogeneity correction. One can already anticipate that it will be concentrated in the same region where the homogeneous correction was significant, i.e. $\beta M<1$ (cf. [5]), being exponentially suppressed for higher values of the field. In fact, a numerical study of the complete $W_{q}$ shows that this function is peaked around $\phi=0$ and non-negligible for $\beta|\phi|<1$ [14].

The Euler-Lagrange equation for the chiral field up to this order in the gradient expansion reads

$$
\nabla^{2} \phi=\left[1+g W_{q}\left(T, \mu_{q}, \phi\right)\right]^{-1} \frac{\partial V_{e f f}\left(T, \mu_{q}, \phi\right)}{\partial \phi} \equiv V_{i n}^{\prime}(\phi) .
$$

The complete new effective potential can be obtained from our previous results by numerical integration. In order to proceed analytically, though, we choose to fit its derivative, which we know exactly up to this order, by a polynomial of the fifth degree. Actually, we know that the commonly used effective potential, $V_{e f f}=V+V_{q}$, can hardly be distinguished from a fit with a polynomial of sixth degree in the region of interest for nucleation $[5,16]$. Working with fits will be most convenient for using well-known results in the thin-wall approximation to estimate physical quantities that are relevant for nucleation, such as the surface tension and the free energy of the critical bubble [17].

We can now integrate analytically the polynomial approximation to the derivative of the complete effective potential. In Fig. 1 we display the curves for $V_{\text {eff }}$ and $V_{\text {in }}$ for a few values 
of temperature and $\mu_{q}=0$. From the figure one can notice a few consequences of the inhomogeneity correction. A first general effect is the smoothening of the effective potential. In particular, and most importantly, the barrier between the symmetric phase and the broken phase is significantly diminished, as well as the depth of the broken phase minimum. Therefore, one can expect an augmentation in the bubble nucleation rate. In principle, one should also have better results from calculations within the thin-wall approximation. Also, the critical temperature moves up slightly.

Let us now consider the effects of the first inhomogeneity correction on the process of phase conversion driven by the nucleation of bubbles [17]. To work with approximate analytic formulas, we follow Ref. [5] and express $V_{\text {in }}$ over the range $0 \leq \phi \leq T$ in the familiar Landau-Ginzburg form $V_{\text {eff }} \approx \sum_{n=0}^{4} a_{n} \Phi^{n}$. Although this approximation is obviously incapable of reproducing all three minima of $V_{i n}$, this polynomial form is found to provide a good quantitative description of $V_{i n}$ in the region of interest for nucleation, i.e. where the minima for the symmetric and broken phases, as well as the barrier between them, are located. Using the thin-wall approximation, we can compute the relevant quantities in the way described in Ref. [5]. Nevertheless, it was shown in [5] that the thin-wall limit becomes very imprecise as one approaches the spinodal. In this vein, the analysis presented below is to be regarded as semi-quantitative. To be consistent will compare results from the homogeneous calculation to those including the inhomogeneity correction within the same approximation [14].

To illustrate the effect, we compute the critical radius, $R_{c}$, and free energy of the critical bubble in units of temperature, $F_{b} / T$, for three different values of the temperature. For $T=108 \mathrm{MeV}$, corresponding to the spinodal, which is not modified by the first inhomogeneity correction, the corrected values are $R_{c} \approx 0.98 \mathrm{fm}$ and $F_{b} / T \approx 0.20$, as compared to $R_{c} \approx 1.1 \mathrm{fm}$ and $F_{b} / T \approx 0.9$ in the homogeneous case. The same computation for $T=116 \mathrm{MeV}$ yields $R_{c} \approx 2.15 \mathrm{fm}$ and $F_{b} / T \approx 1.14$, as compared to $R_{c} \approx 2.2 \mathrm{fm}$ and $F_{b} / T \approx 2.1$. At $T=123 \mathrm{MeV}$, which corresponds to the critical temperature for the homogeneous case, the critical radius and $F_{b} / T$ diverge in the homogeneous computation, whereas $R_{c} \approx 35 \mathrm{fm}$ and $F_{b} / T \approx 394$ including inhomogeneities. The numbers above clearly indicate that the formation of critical bubbles is much less suppressed in the scenario with inhomogeneities, which will in principle accelerate the phase conversion process after the chiral transition.

In summary, the first inhomogeneity correction brings significant modifications to the form of the effective potential. Besides a general smoothening of the potential, the critical temperature moves upward and the hight of the barrier separating the symmetric and the broken phase vacua diminishes appreciably. As a direct consequence, the radius of the critical bubble goes down, as well as its free energy, and the process of nucleation is facilitated. This effect will compete with dissipation corrections that tend to slow down the process of phase conversion [16]. Although the numbers presented above should be regarded as simple estimates, since they rely on a number of approximations, the qualitative behavior is clear. A more detailed analysis can be found in Ref. [14]

\section{Acknowledgments}

We thank D.G. Barci, H. Boschi-Filho, C.A.A. de Carvalho, T. Kodama, and especially A. Dumitru for fruitful discussions. This work was partially supported by CAPES, CNPq, FAPERJ and FUJB/UFRJ.
[1] L. P. Csernai and I. N. Mishustin, Phys. Rev. Lett. 74, 5005 (1995); A. Abada and J. Aichelin, Phys. Rev. Lett. 74, 3130 (1995); A. Abada and M. C. Birse, Phys. Rev. D 55, 6887 (1997);

[2] I. N. Mishustin and O. Scavenius, Phys. Rev. Lett. 83, 3134 (1999).

[3] O. Scavenius and A. Dumitru, Phys. Rev. Lett. 83, 4697 (1999).

[4] O. Scavenius, A. Mocsy, I. N. Mishustin, and D. H. Rischke, Phys. Rev. C 64, 045202 (2001).

[5] O. Scavenius, A. Dumitru, E. S. Fraga, J. T. Lenaghan, and A. D. Jackson, Phys. Rev. D 63, 116003 (2001).

[6] K. Paech, H. Stoecker, and A. Dumitru, Phys. Rev. C 68, 044907 (2003); K. Paech and A. Dumitru, Phys. Lett. B 623 200 (2005).

[7] C. E. Aguiar, E. S. Fraga, and T. Kodama, J. Phys. G 32, 179 (2006).

[8] R. D. Pisarski, Phys. Rev. D 62, 111501 (2000); A. Dumitru and R. D. Pisarski, Phys. Lett. B 504, 282 (2001); A. Dumitru and R. D. Pisarski, Nucl. Phys. A 698, 444 (2002).

[9] O. Scavenius, A. Dumitru, and A. D. Jackson, Phys. Rev. Lett. 87, 182302 (2001).
[10] L. P. Csernai and J. I. Kapusta, Phys. Rev. D 46, 1379 (1992)

[11] M. Gell-Mann and M. Levy, Nuovo Cim. 16, 705 (1960); R. D. Pisarski, Phys. Rev. Lett. 76, 3084 (1996).

[12] J. Kapusta, Finite Temperature Field Theory (Cambridge University Press, Cambridge, 1989).

[13] A. Dumitru, L. Portugal, and D. Zschiesche, nucl-th/0502051; Phys. Rev. C 73, 024902 (2006).

[14] B. G. Taketani and E. S. Fraga, to appear

[15] T. D. Lee and M. Margulies, Phys. Rev. D 11, 1591 (1975) [Erratum-ibid. D 12, 4008 (1975)]; A. Bochkarev and J. I. Kapusta, Phys. Rev. D 54, 4066 (1996); G. W. Carter, P. J. Ellis, and S. Rudaz, III: Mesons Nucl. Phys. A 618, 317 (1997); A. Mocsy, I. N. Mishustin, and P. J. Ellis, Phys. Rev. C 70, 015204 (2004).

[16] E. S. Fraga and G. Krein, Phys. Lett. B 614, 181 (2005); E. S. Fraga, hep-ph/0510344.

[17] J. D. Gunton, M. San Miguel, and P. S. Sahni, in Phase Transitions and Critical Phenomena (Eds.: C. Domb and J. L. Lebowitz, Academic Press, London, 1983), v. 8. 